\title{
Optimal Blurred Segments Decomposition in Linear Time
}

\author{
Isabelle Debled-Rennesson ${ }^{1}$, Fabien Feschet ${ }^{2}$, and Jocelyne Rouyer-Degli ${ }^{1}$ \\ 1 LORIA Nancy - Campus Scientifique - BP 239, \\ 54506 Vandœuvre-lès-Nancy Cedex \\ \{debled, rouyer\}@loria.fr \\ 2 LLAIC - IUT Clermont-Ferrand - Campus des Cézeaux, \\ 63172 Aubière Cedex - France \\ feschet@llaic3.u-clermont1.fr
}

\begin{abstract}
Blurred (previously named fuzzy) segments were introduced by Debled-Rennesson et al [1,2] as an extension of the arithmetical approach of Reveillès [1] on discrete lines, to take into account noise in digital images. An incremental linear-time algorithm was presented to decompose a discrete curve into blurred segments with order bounded by a parameter $d$. However, that algorithm fails to segment discrete curves into a minimal number of blurred segments. We show in this paper, that this characteristic is intrinsic to the whole class of blurred segments. We thus introduce a subclass of blurred segments, based on a geometric measure of thickness. We provide a new convex hull based incremental linear time algorithm for segmenting discrete curves into a minimal number of thin blurred segments.
\end{abstract}

\section{Introduction}

Discrete (also called digital) segments are well known objects which have been thoroughly studied for more than 30 years [12. There are many definitions of discrete segments, all equivalent for 8-connected discrete sets and 4-connected discrete sets. Discrete segments serve as building blocks for representation [16], decomposition [4 or analysis of discrete curves and more generally shapes. For instance, polygonalizations of discrete curves are widely used in shape representation [16] and can be computed in linear time [14, 3, 7]. Moreover, the use of discrete segments permits a perfect representation or reconstruction of discrete curves. However, this might result in complicated representations when discrete curves include noise or have been distorted by an acquisition process. Many polygonal approximation methods have been proposed throughout the years using different approachs [9, 13, 15, 6]. To deal with noise and as an extension of the results presented in [3], the notion of fuzzy segments was introduced in [1,2]. From now on, we shall name these segments blurred segments rather than fuzzy segments in order to prevent any confusion with fuzzy logic and fuzzy geometry. The theorem of Debled-Rennesson and Reveillès [3] provides an incremental algorithm with linear-time complexity for the recognition of discrete segments using 
arithmetical properties and has been extended to the case of blurred segments by Debled-Rennesson et al 11. However, blurred segments represent supersets of the original discrete data and thus, the result obtained with the previous theorem can not be guaranteed to be optimal, in the sense that the orders of the blurred segments are not necessarily minimal. We present in this paper a study of the order of blurred segments and point out the reason why it is difficult to mimimize the order of a blurred segment in the recognition process. Hence, we present theoretical arguments to justify a restriction in the class of blurred segments in order to guarantee optimality in the recognition process. Moreover, our approach can deal with disconnected sets which was impossible with the theorem given in 1 .

The paper is organized as follows. In section 2 we recall definitions and properties used in 11. We present in section 2.3, a problem in the minimization of the order of recognized blurred segments. This problem is explained by theorem 2 and leads to the introduction of a subclass of blurred segments by adding a geometric characterization based on convex hulls. A new recognition algorithm of blurred segments is described in section 3 by the study of their equivalent characterizations in terms of convex hulls. An incremental linear-time recognition algorithm is presented which guarantees that the computed blurred segments are the thinnest possible ones. Experiments are given in section 4 to show the quality of the decomposition of the proposed algorithm. The paper ends up with some conclusions and perspectives in section 5 .

\section{Blurred Segments}

\subsection{Definitions}

The notion of blurred (also called fuzzy) segments relies on the arithmetical definition of discrete lines 11 where a line, with slope $\frac{a}{b}$, lower bound $\mu$ and thickness $\omega$ (with $a, b, \mu$ and $\omega$ being integer such that $\operatorname{gcd}(a, b)=1$ ) is the set of integer points $(x, y)$ verifying $\mu \leq a x-b y<\mu+\omega$. Such a line is denoted by $\mathcal{D}(a, b, \mu, \omega)$. The real lines $a x-b y=\mu+\omega-1$ and $a x-b y=\mu$ are respectively named the upper and lower leaning lines of $\mathcal{D}(a, b, \mu, \omega)[3$. The integer points $\left(x_{L}, y_{L}\right)$ (resp. $\left.\left(x_{U}, y_{U}\right)\right)$ of the lower (resp. upper) leaning lines of $\mathcal{D}(a, b, \mu, \omega)$ are called the lower (resp. upper) leaning points of $\mathcal{D}(a, b, \mu, \omega)$. We refer to Fig. 1 for a descriptive example of these notions.

In the following, we restrict our study to points of the first octant of $\mathbb{Z}^{2}$, due to symmetries with respect to $O x, O y$ and the real line $x=y$. We thus always have $0 \leq y \leq x$. This hypothesis can be done without loss of generality and simplifies notations, proofs and definitions.

Definition 1. [1] A set $\mathcal{S}_{b}$ of consecutive points $\left(\left|\mathcal{S}_{b}\right| \geq 2\right)$ of an 8-connected curve is a blurred segment with order $\boldsymbol{d}$ if there is a discrete line $\mathcal{D}(a, b, \mu, \omega)$, called bounding, such that all points of $\mathcal{S}_{b}$ belong to $\mathcal{D}$ and $\frac{\omega}{\max (|a|,|b|)} \leq d$.

The notion of order of a blurred segment has been introduced to make a difference compared to the thickness of bounding lines of $\mathcal{S}$, since any sufficiently 


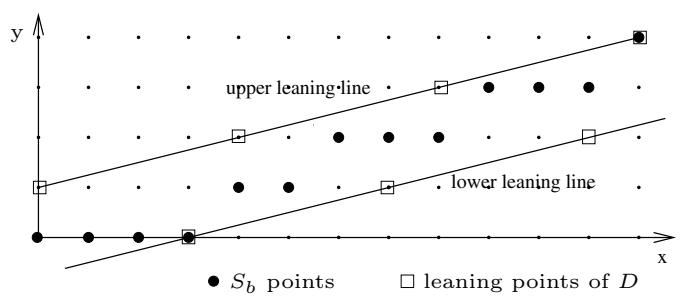

Fig. 1. A strictly bounding line $D$ of a blurred segment $S_{b}$

thick discrete line can contain $\mathcal{S}$. Thus, two discrete lines containing $\mathcal{S}$ can be compared with respect to their orders and this leads to a classification of the lines containing $\mathcal{S}$. To be reasonably closed to the points of $\mathcal{S}$, more restrictive conditions onto the discrete lines containing $\mathcal{S}$ must be introduced as follows.

Definition 2. [1] Let $\mathcal{S}_{b}$ be a blurred segment with order d whose abscissa interval is $[0, l-1]$ and let $\mathcal{D}(a, b, \mu, \omega)$ be a bounding line of $\mathcal{S}_{b}$. D is named strictly bounding for $\mathcal{S}_{b}$ if $\mathcal{D}$ possesses at least three leaning points in the interval $[0, l-1]$ and, $\mathcal{S}_{b}$ contains at least one lower leaning point and one upper leaning point of $\mathcal{D}$.

In Fig. 1 $\mathcal{D}(1,4,-4,8)$ is a strictly bounding line of the blurred segment $S_{b}$. The leaning points of $\mathcal{D}$ are the points $(4 k+3, k)$ and $(4 k, k+1)$, for $k \in[0,3]$ and $S_{b}$ contains the lower leaning point $(3,0)$ and the upper leaning point $(12,4)$.

\subsection{Algorithm for Segmentation into Blurred Segments with Strictly Bounding Lines}

We briefly recall in this paragraph the technique used in [1] to segment a discrete curve into order $d$ blurred segments with strictly bounding lines. This segmentation relies on the following theorem, which studies the different possible cases of the growth of a blurred segment.

Theorem 1. 11 Let us consider a blurred segment $\mathcal{S}_{b}$ in the first octant whose abscissa interval is $[0, l-1]$ and $\mathcal{D}(a, b, \mu, \omega)$, a strictly bounding line. In this case, the order of $\mathcal{S}_{b}$ is $\frac{\omega}{b}$. Let $M\left(x_{M}, y_{M}\right)$ be an integer point connected to $\mathcal{S}_{b}$ whose abscissa is equal to $l$ or $l-1$. Let the remainder at $M$, denoted $r(M)$, be a function of $\mathcal{D}$ defined as $\mathbf{r}(\mathbf{M})=\mathbf{a x}_{\mathbf{M}}-\mathbf{b y}_{\mathbf{M}}$.

(i) If $\mu \leq r(M)<\mu+\omega$, then $M \in \mathcal{D}$;

$\mathcal{S}_{b} \cup M$ is a blurred segment with order $\frac{\omega}{b}$ and with $\mathcal{D}$ as strictly bounding line.

(ii) If $r(M) \leq \mu-1$, then $M$ is external to $\mathcal{D}$;

$\mathcal{S}_{b} \cup M$ is a blurred segment with order $\frac{\omega^{\prime}}{b^{\prime}}$ and the line $\mathcal{D}^{\prime}\left(a^{\prime}, b^{\prime}, \mu^{\prime}, \omega^{\prime}\right)$ is strictly bounding, with

- $b^{\prime}$ and $a^{\prime}$ coordinates of the vector $\overrightarrow{P_{r(M)+1} M}, P_{r(M)+1}$ being the point whose remainder is $r(M)+1$ with respect to $\mathcal{D}$ and $x_{P_{r(M)+1}} \in[0, b-1]$, 

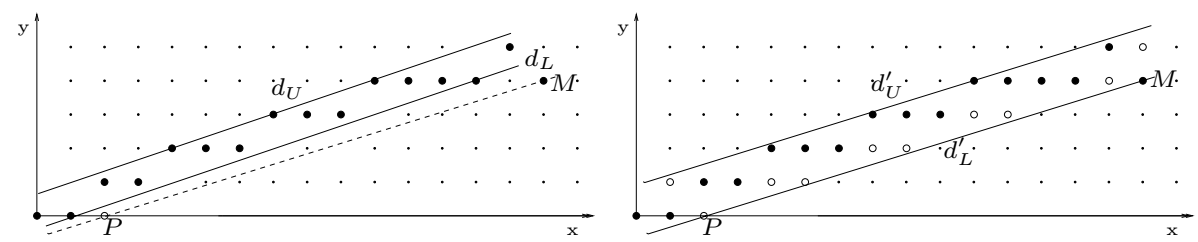

Fig. 2. An example of blurred segment growth relying on Theorem 1

$-\mu^{\prime}=a^{\prime} x_{M}-b^{\prime} y_{M}$

$-\omega^{\prime}=a^{\prime} x_{L_{L}}-b^{\prime} y_{L_{L}}-\mu^{\prime}+1$, with $L_{L}\left(x_{L_{L}}, y_{L_{L}}\right)$ last lower leaning point of the line $\mathcal{D}$ present in $\mathcal{S}_{b}$.

(iii) If $r(M) \geq \mu+\omega$, then $M$ is external to $\mathcal{D}$;

$\mathcal{S}_{b} \cup\{M\}$ is a blurred segment with order $\frac{\omega^{\prime}}{b^{\prime}}$ and the line $\mathcal{D}^{\prime}\left(a^{\prime}, b^{\prime}, \mu^{\prime}, \omega^{\prime}\right)$ is strictly bounding with

- $b^{\prime}$ and $a^{\prime}$ coordinates of the vector $\overrightarrow{P_{r(M)-1} M}, P_{r(M)-1}$ being the point whose remainder is $r(M)-1$ with respect to $\mathcal{D}$ and $x_{P_{r(M)-1}} \in[0, b-1]$,

$-\mu^{\prime}=a^{\prime} x_{U_{L}}-b^{\prime} y_{U_{L}}$ with $U_{L}\left(x_{U_{L}}, y_{U_{L}}\right)$ last upper leaning point of the line $\mathcal{D}$ present in $\mathcal{S}_{b}$,

$-\omega^{\prime}=a^{\prime} x_{M}-b^{\prime} y_{M}-\mu^{\prime}+1$.

An example of application of this theorem is given in Fig. 2. A blurred segment $\mathcal{S}_{b}$ with order 2 is depicted in Fig. 2 (left). $\mathcal{D}(1,3,-2,4)$ is strictly bounding for $\mathcal{S}_{b}, d_{U}$ and $d_{L}$ are the leaning lines of $\mathcal{D}$. The point $M(15,4)$ is added to $\mathcal{S}_{b}$. Since $r(M)=3$, adding $M$ to $\mathcal{S}_{b}$ corresponds to the case (iii) of the theorem: $P$ is the point in $[0,2]$ such that $r(P)=2$, the slope of $\mathcal{D}^{\prime}$ is computed with the vector $P M$, therefore $\mathcal{D}^{\prime}(4,13,-12,21)$ is strictly bounding for $\mathcal{S}_{b} \cup\{M\}$. In Fig. 2 (right), a representation of $\mathcal{D}^{\prime}$ and $\mathcal{S}_{b} \cup\{M\}$ (black points) is given. The points of $\mathcal{D}^{\prime}$ which do not belong to $\mathcal{S}_{b} \cup\{M\}$ are in white, $d_{U}^{\prime}$ and $d_{L}^{\prime}$ are the leaning lines of $\mathcal{D}^{\prime}$.

A linear time incremental algorithm of segmentation into order $d$ blurred segments was deduced from this theorem in [1,2]. The principle was as follows: let $\mathcal{S}_{b}$ be the current order $d$ blurred segment, a point $M$ of $\mathcal{C}$ was added to $\mathcal{S}_{b}$, the characteristics of a strictly bounding line of $\mathcal{S}_{b} \cup M$ were computed according to Theorem 1. The current segment included the point $M$ if the value of the obtained ratio $\omega / \max (|a|,|b|)$ was lower than or equal to the order $d$. Else, the current order $d$ blurred segment ended at the point located before $M$ in $\mathcal{C}$ and a new order $d$ blurred segment started at $M$.

\subsection{Main Drawback}

Theorem 1 describes an incremental method to construct a strictly bounding line. However the constructions do not garantee that the order of the built bounding line is minimal. Hence, the segmentation of a discrete curve might be done into too many blurred segments. For instance, the curve depicted in Fig. 3 (left) would be uncorrectedly segmented into several parts with $d=1.9$, while there exists a 

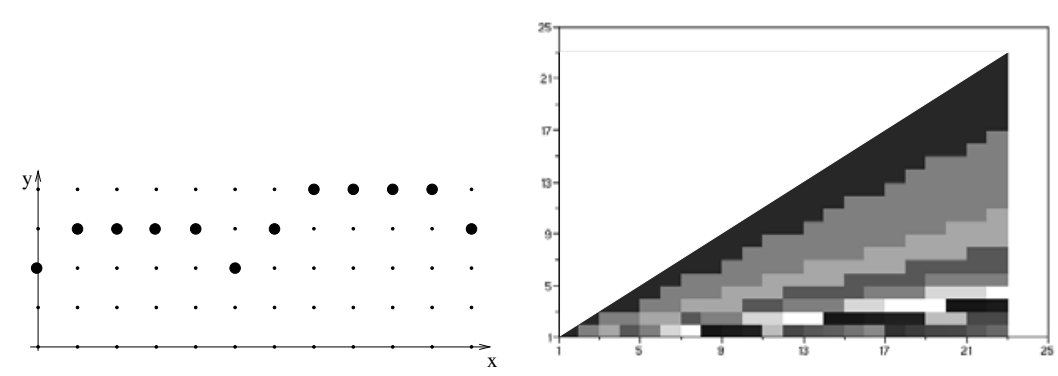

Fig. 3. (left) a sample discrete curve (right) gray scale plot of $\omega / b$ with $b$ in $x$-axis and $a$ in y-axis

bounding line with ratio strictly lower than 1.9. The experimental investigations that we have performed, induced us to study in detail a better measure for segment thickness to garantee the order of the built bounding lines.

To understand the ratio $\omega / \max (|a|,|b|)$, we study it in the first octant using the curve in Fig. 3. For any $a$ and $b$ values, with $a \leq b$, we compute the remainder $r(M)$. The value of $\omega$ is obtained by adding 1 to the difference between the maximum and the minimum of the remainders. We denote by $\omega(a, b)$ this value. The result is depicted on Fig. 3 (right) where light grey values correspond to low values of the ratio $\omega / b$. As shown in Fig. 3 (right), the function $\omega(a, b) / b$ has a lot of local extrema. The minimalization of $\omega(a, b) / b$ seems to be a hard combinatorial problem. Moreover, $\omega(a, b) / b$ is sensitive to multiplications since the couples $(a, b)$ equal to $(1,6),(3,18)$ and $(4,24)$ do not produce the same value. Their values are respectively $1.83,1.72$ and 1.70 . The following theorem given for the first octant without loss of generality explains why the minimization of the ratio $\omega(a, b) / b$ might be impossible to obtain due to its asymptotic behaviour.

Theorem 2. For any finite set $\mathcal{S}_{b}$, let us denote by $\mathcal{W}$ the series $\left(\omega\left(a_{k}, b_{k}\right) / b_{k}\right)$ where $k$ is a positive integer, $a_{k}=k$ and $b_{k}=k b_{0}+\lambda$, with $b_{0}$ and $\lambda$ positive integers. Then $\mathcal{W}$ is decreasing and has a limit equals to $\frac{\omega\left(1, b_{0}\right)-1}{b_{0}}$.

Proof.

We introduce the remainder $r_{(a, b)}(M)=a x_{M}-b y_{M}$. It is easy to see that $r_{\left(a_{k}, b_{k}\right)}(M)=k r_{\left(1, b_{0}\right)}(M)-\lambda y_{M}$. We now introduce $\Delta_{(a, b)}\left(M, M^{\prime}\right)$ as follows,

$$
\Delta_{\left(a_{k}, b_{k}\right)}\left(M, M^{\prime}\right)=r_{(a, b)}(M)-r_{(a, b)}\left(M^{\prime}\right)=k \Delta_{\left(1, b_{0}\right)}\left(M, M^{\prime}\right)+\lambda\left(y_{M^{\prime}}-y_{M}\right)
$$

Suppose that $\Delta_{\left(1, b_{0}\right)}\left(M, M^{\prime}\right)=0$. Hence, $\Delta_{\left(a_{k}, b_{k}\right)}\left(M, M^{\prime}\right)=\lambda\left(y_{M^{\prime}}-y_{M}\right)$. Since $\left(y_{M^{\prime}}-y_{M}\right)$ is bounded on any finite set and $\lambda$ is constant, we deduce that the previous value is bounded above by a constant $\delta$.

Suppose now that $\Delta_{\left(1, b_{0}\right)}\left(M, M^{\prime}\right) \neq 0$. By using the same boundedness argument, we see that there exists a value $k_{0}$ such that

$$
\Delta_{\left(a_{k}, b_{k}\right)}\left(M, M^{\prime}\right)>0(\text { resp. }<0) \Longleftrightarrow \Delta_{\left(1, b_{0}\right)}\left(M, M^{\prime}\right)>0(\text { resp. }<0)
$$


for any $k \geq k_{0}$. Hence asymptoticaly, the remainders $r_{\left(a_{k}, b_{k}\right)}($.$) and the remain-$ ders $r_{\left(1, b_{0}\right)}($.$) have the same ordering. However, the remainders r_{\left(a_{k}, b_{k}\right)}($.$) are$ diverging when $k$ tends to infinity. Thus for sufficiently large $k$, the minimum and the maximum of the remainders $r_{\left(a_{k}, b_{k}\right)}($.$) are obtained exactly for the same$ points $M_{\min }$ and $M_{\max }$ as for the remainders $r_{\left(1, b_{0}\right)}($.$) . This permits us to deduce$ that for sufficiently large $k$,

$$
\omega_{\left(a_{k}, b_{k}\right)}=k\left(\omega_{\left(1, b_{0}\right)}-1\right)+\lambda\left(y_{M_{\min }}-y_{M_{\max }}\right)+1
$$

So,

$$
\frac{\omega_{\left(a_{k}, b_{k}\right)}}{b_{k}}=\frac{k\left(\omega_{\left(1, b_{0}\right)}-1\right)}{k b_{0}+\lambda}+\frac{y_{M_{\min }}-y_{M_{\max }}+1}{k b_{0}+\lambda}
$$

The limits of the previous expression is given by the limit of the first term, specifically

$$
\lim _{k \rightarrow+\infty} \frac{\omega_{\left(a_{k}, b_{k}\right)}}{b_{k}}=\frac{\left(\omega_{\left(1, b_{0}\right)}-1\right)}{b_{0}}
$$

We conclude the proof by a study of a specific case obtained when the remainders $r\left(1, b_{0}\right)$ are all equals. In such a case, $\omega\left(1, b_{0}\right)=1$ and $\omega\left(a_{k}, b_{k}\right)=$ $1+\lambda\left(\max _{M} y_{M}-\min _{M} y_{M}\right)$. Thus by dividing by $b_{k}$ and taking the limit, we obtain 0 . The result still holds and this concludes the proof.

To have an optimal algorithm, we slightly modify the subclass of considered blurred segments by taking the limit measure of the previous theorem as a measure for comparing blurred segments.

We start by giving a geometric description of the limit measure. The vertical distance of a discrete line $\mathcal{D}(a, b, \mu, \omega)$ is the vertical distance (ordinate difference) between the leaning lines of $\mathcal{D}$ and is equal to $\frac{\omega-1}{b}$. We now recall that a supporting line of a convex set $C$ is a line $l$ such that the intersection of $l$ with $C$ is not empty, and such that $C$ is entirely either below or above $l[5$. The vertical distance of a convex set $C$, is the minimal vertical distance of any pair of parallel supporting lines. This could also be defined as the maximal length of the intersection of $C$ with a vertical line.

Definition 3. Let us consider a set of 8-connected points $\mathcal{S}_{b}$. A bounding line of $\mathcal{S}_{b}$ is said optimal if its vertical distance is minimal, i.e. if the vertical distance of $\mathcal{S}_{b}$ equals to the vertical distance of its convex hull conv $\left(\mathcal{S}_{b}\right)$.

This definition is illustrated in Fig. 4 and leads to the following new definition concerning blurred segments.

Definition 4. $A$ set $\mathcal{S}_{b}$ is a blurred segment of width $\nu$ if and only if its optimal bounding line has a vertical distance less or equal to $\nu$.

The recognition of blurred segments with width $\nu$ is thus equivalent to the computation of the vertical distance of the convex set $\operatorname{conv}\left(\mathcal{S}_{b}\right)$. 


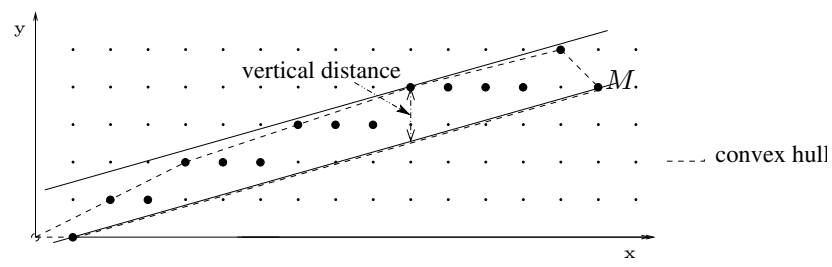

Fig. 4. An optimal bounding line

\section{Convex Hulls and Blurred Segments}

\subsection{Characterization of the Vertical Distance of a Finite Convex Set}

Let us denote by $L_{i}$ and $U_{i}$ respectively the lowest and highest intersection point of a finite convex set $C$ with the vertical line given by $x=i$ and by $\mathcal{V}(i)$ the distance between $L_{i}$ and $U_{i}$, then for any $i \neq j$, the quadrangle $\left(L_{j} U_{j} U_{i} L_{i}\right)$ is located inside $C$. It is straightforward to see that the function $\mathcal{V}($.$) is a concave$ function. Hence, every local maximum of the function is also a global maximum of the function. The positions of maxima of the function $\mathcal{V}($.$) have the following$ property.

Proposition 1. For any finite convex set $C$, the function $\mathcal{V}($.$) has a maximum$ value at a position $i$ where $L_{i}$ or $U_{i}$ can be chosen to be a vertex of the border, $B d C$, of $C$.

Proof. Let us consider a position $i$ such that neither $L_{i}$ nor $U_{i}$ are vertices of $\mathrm{Bd} C$ and the edges of $\mathrm{Bd} C$ containing $L_{i}$ and $U_{i}$. It is easy to see that $i$ cannot be the position of a maximum when the slopes of these edges are not equal. If the case of equality we can move on the edges until one of the two points becomes a vertex of $\mathrm{Bd} C$.

From this proposition, we can deduce two facts if we are looking for the vertical distance of a convex set: first, we only have to consider points on the border of $C$ and second, extrema are obtained for some $x$ positions of the vertices of $\mathrm{Bd} C$. This leads to three cases: (edge,vertex), (vertex,edge) or (vertex,vertex) where the first element represents the lower part of the convex set and the second element represents the upper part. It must be noticed that the previous proposition is identical to the one characterizing the width of a convex set [10].

It is clear that the edges corresponding to the position of a maximum of $\mathcal{V}($.$) are supporting lines, taking horizontal lines for a couple (vertex,vertex).$ Moreover, the edge and its parallel passing through the vertex define exactly the lower and upper leaning lines of an optimal bounding line for $C$. Hence, optimal lines are deduced from the positions of maxima. 

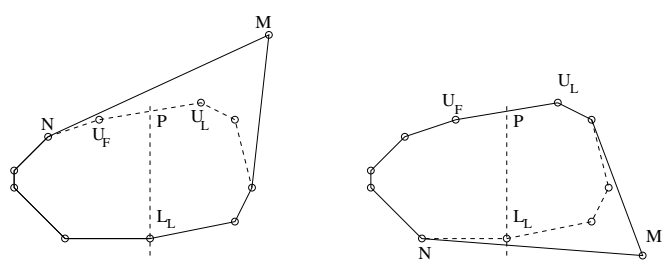

Fig. 5. Adding a new point either above (left) or below (right)

\subsection{New Recognition Algorithm}

Let $\mathcal{S}_{b}=\left\{\left(x_{i}, y_{i}\right), 0 \leq i<n\right\}$, a blurred segment in the first octant with $\mathcal{D}(a, b, \mu, \omega)$ as optimal bounding line. We suppose that $\mathcal{S}_{b}$ contains two upper leaning points, $U_{F}$ and $U_{L}$, and one lower leaning point, $L_{L} . U_{F}, U_{L}$ and $L_{L}$ are vertices of the convex hull of $\mathcal{S}_{b} \operatorname{conv}\left(\mathcal{S}_{b}\right)$. Moreover, the vertical distance of $\mathcal{D}$ and of $\operatorname{conv}\left(\mathcal{S}_{b}\right)$ can be calculated at the point $L_{L}$. To compute the convex hull of $\mathcal{S}_{b}$, we use Melkman's algorithm [8]. Let us recall that this algorithm incrementally computes the convex hull of $n$ points forming a simple polygonal line in $O(n)$ time based on a double ended queue (deque) list. Since points are added with increasing $x$, we are guaranteed to have a simple polygonal line.

Growth of a Blurred Segment. Suppose that we add a new point $M$ to $\mathcal{S}_{b}$, $\mathcal{S}_{b}^{\prime}=\mathcal{S}_{b} \cup\{M\}$. There are three cases: $M$ is added above, inside or below $\mathcal{D}$.

If $M$ belongs to $\mathcal{D}$ then, after the application of Melkman's algorithm 8 , the vertical distance remains the same. The new set of points, $\mathcal{S}_{b}^{\prime}$, is a blurred segment with the same width and with the same optimal bounding line $\mathcal{D}$. In the other cases, the vertical distance must be recomputed. $P$, the intersection point between the vertical line from $L_{L}$ and $U_{F} U_{L}$, is strictly inside $\left[U_{F} U_{L}\right]$ (see Fig. (5).

Suppose that $M$ is added above $\mathcal{D}$. Let us apply Melkman's algorithm. The convex set is modified and we call $N$ the point before $M$ in the upper part of the resulting convex hull, see Fig. 5 (left). $N$ is necessarily before $U_{F}$ or is $U_{F}$. As a consequence, the vertical projection of $L_{L}$ is inside $[N M]$. Thus, the vertical distance of the new convex set strictly increases. The key point is to locate the new position of a maximum. It is clear that $N$ cannot represent a position of a maximum since $\mathcal{V}(N) \leq \mathcal{V}\left(L_{L}\right)$. Moreover, $M$ does not project vertically strictly inside an edge of the lower part of $\operatorname{conv}\left(\mathcal{S}_{b}^{\prime}\right)$. Hence, the new position of a maximum if necessarily obtained for one point at the right of $L_{L}$ in the lower part of $\operatorname{conv}\left(\mathcal{S}_{b}^{\prime}\right)$. Let us recall now that local extrema are global extrema for the function $\mathcal{V}($.$) such that we only have to test the candidate points in sequence$ and stop at the first local maximum, called $C$.

Suppose now that $M$ is added below $\mathcal{D}$. Let us apply Melkman's algorithm. The lower part of the convex set is modified and we still call $N$ the point before $M$ in the lower part of the new convex hull, see Fig. 5 (right). $N$ is necessarily to the left of $L_{L}$. Hence, $N$ cannot be the new position of a maximum since $\mathcal{V}(N) \leq \mathcal{V}\left(L_{L}\right)$. We do not know precisely where $N$ is located in comparison 
with $\left[U_{F} U_{L}\right]$. It might be either on the left of $U_{F}$ or inside $\left[U_{F} U_{L}\right]$. In both cases, it is however straightforward to see that no position strictly to the left of $U_{L}$ can be the position of a maximum. As in the previous case, neither $N$ nor $M$ can be positions of maxima. So, the new position of a maximum is given by one point situated to the right of $U_{L}$ on the upper part of the new convex hull and we only have to test the candidate points in sequence and stop at the first local maximum, called $C$.

In both these cases, $\mathcal{S}_{b}^{\prime}$ is a blurred segment with optimal bounding line $\mathcal{D}^{\prime}$ for which the points $M, N$, and $C$ are leaning points. Moreover, the vertical distance of $\operatorname{conv}\left(\mathcal{S}_{b}^{\prime}\right)$ is equal to the vertical distance of $\mathcal{D}^{\prime}$ and can be calculated at the point $C$.

We have neglected the case where $L_{L}$ and $P$ are vertices of $\operatorname{conv}\left(\mathcal{S}_{b}\right)$. If we choose any edge containing either $L_{L}$ or $P$ and keep the other point as a vertex, we obtain a couple (vertex,edge) or (edge,vertex) with the same vertical distance. This result is of course different from the one of the width of a convex set [5], but applies perfectly in our context.

Recognition Algorithm. From the study of the growth of a blurred segment we can deduce an incremental recognition algorithm of width $\nu$ blurred segments (see Algorithm 1) where points are taken in order of increasing $x$ values. It gives as result a boolean value equal to true if a sequence of points $S$ (input) is a width $\nu$ blurred segment. Morever the last calculated values of $a, b, \mu$ and $\omega$ are the characteristics of an optimal bounding line of $S$.

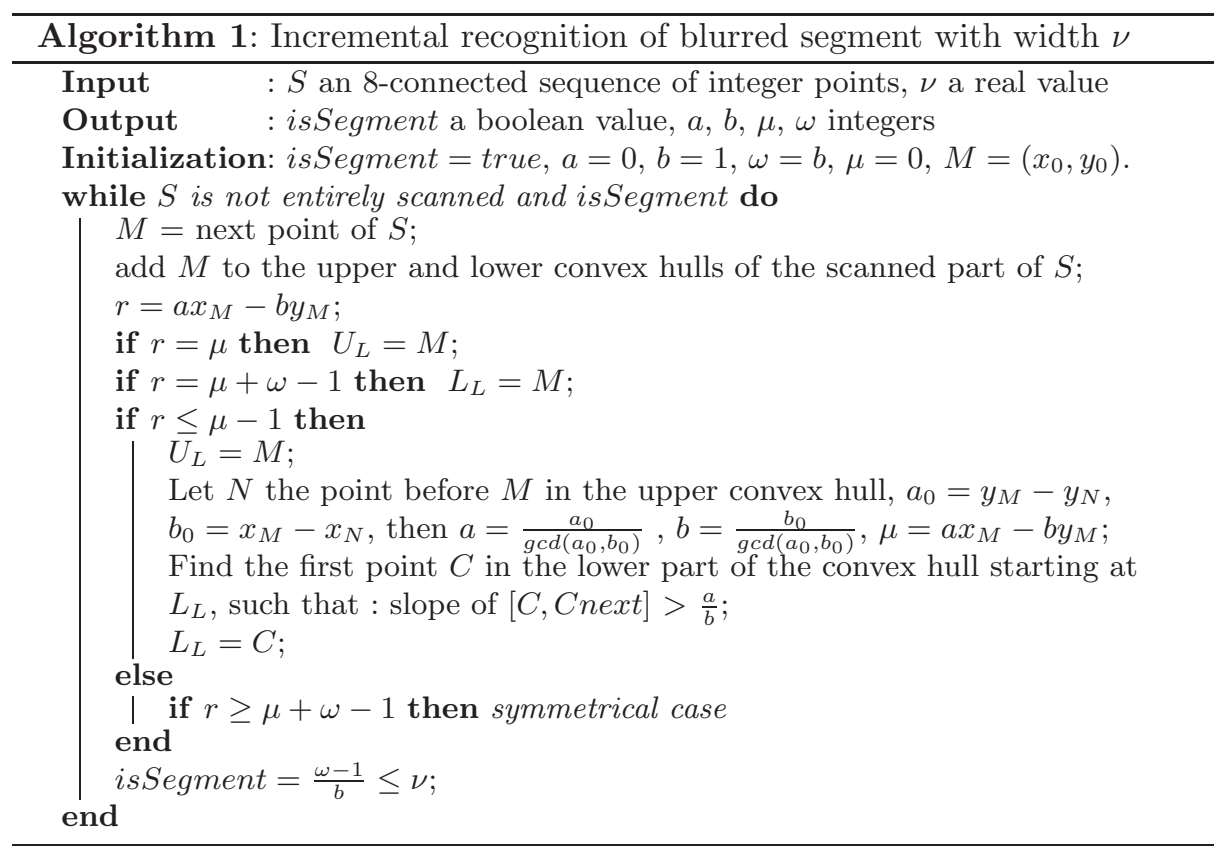



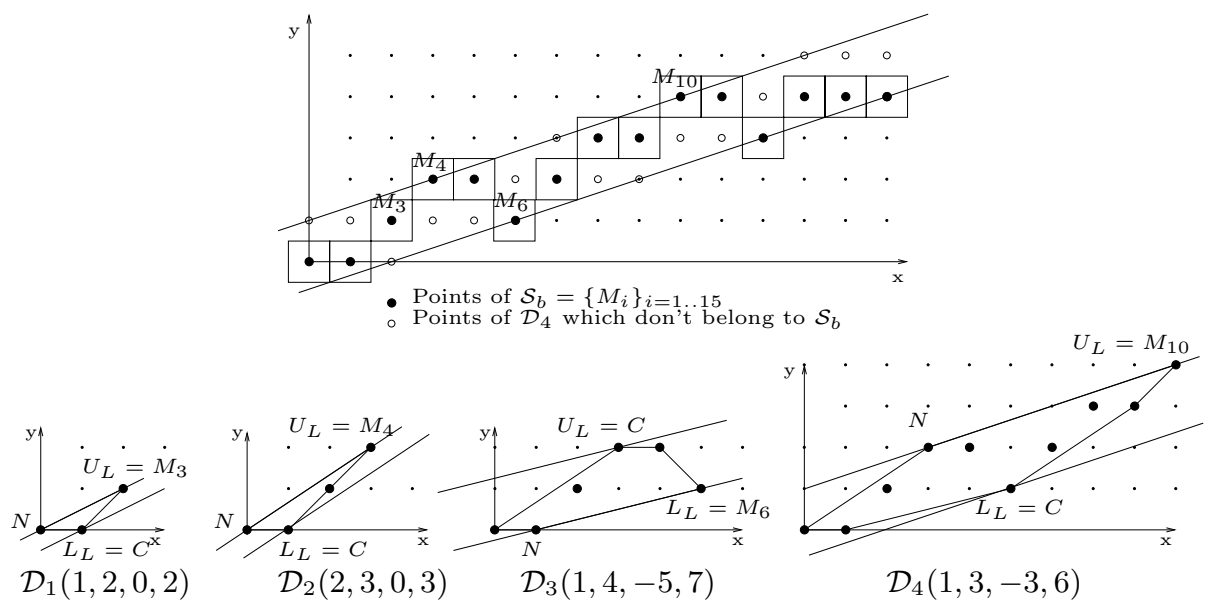

Fig. 6. An example of width 2 blurred segment incremental recognition

Complexity. We now study the complexity of Algorithm 1 on a set $\mathcal{S}$ of $n$ points. The first part of the algorithm is the update of the convex hull after the insertion of the new point $M$. However, Melkman's algorithm 8 has a lineartime complexity for the whole set of insertions. Since, a point is used only for one convex hull, we deduce easily that this part still have a linear-time complexity. The second part of the algorithm concerns the update of the position of a maximum. Each time a point is added, part of the previous convex hull is examined to detect the new position of a maximum. However, the main property of the function $\mathcal{V}($.$) is that it is a concave function. Thus as previously mentioned, we$ only test points for which $\mathcal{V}($.$) is not locally maximal and stop at the first local$ maximum. This part of the algorithm also has a linear-time complexity. Then we can conclude that Algorithm 1 has a linear-time complexity.

An example of the algorithm processing is depicted in Fig. 6. Part of a discrete curve, $\mathcal{S}_{b}$, is drawn at the top of the figure and the different optimal bounding lines, obtained during the incremental recognition, are given. As it can be seen, there are only four steps in the recognition process and the slopes of the supporting lines decrease or increase with respect to the added points. $\mathcal{S}_{b}$ is a blurred segment of width 2 with $\mathcal{D}_{4}(1,3,-3,6)$ as optimal bounding line.

\section{Experiments}

The segmentation of a curve into blurred segments of width $\nu$ is done incrementally as described in [1,2], and as recalled in section 2.2. When the width of the current segment becomes strictly greater than $\nu$, a blurred segment ends at the previous point, and a new segment is initialised. In Fig. 7 we show how the curves on the left are segmented into blurred segments with width 2 . The curve in Fig. 7 (top) is segmented into 18 segments. The curve in Fig. 7 (bottom) is 


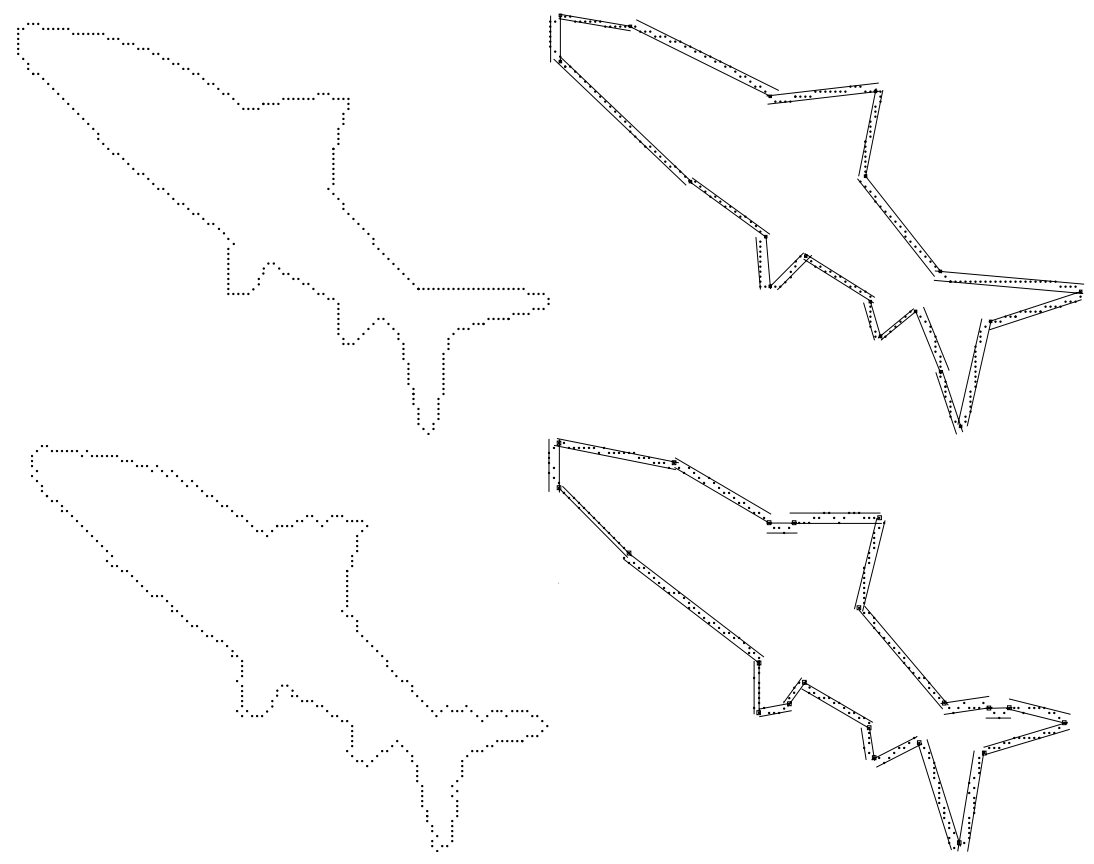

Fig. 7. Shark curves (top without noise, bottom with noise) decomposed into blurred segments of width 2

a noisy version of the top one; the segmentation gives more segments: 21 . If we increase the width of the blurred segments, the number of segments decreases: 19 segments for the width 2.5, 18 segments for the width 3 .

\section{Conclusion}

We have pursued in this paper the study of blurred segments and their applications to discrete noisy curves. Previous works of Debled-Rennesson et al [1] were not able to decompose discrete noisy curves with minimally thin blurred segments. We have identified the origin of this drawback and based on our theoretical study, we have proposed to restrict the class of blurred segments by adding a geometrical bound on their thickness. A proper measure to control the thickness of blurred segments bounded by a discrete line $\mathcal{D}(a, b, \mu, \omega)$ was demonstrated to be $(\omega-1) / b$. Based on this modification, the recognition of blurred segments was shown to be equivalent to the computation of the vertical distance of the convex set of points of the discrete curves. We have presented an incremental linear time algorithm which solves this problem with minimality in the thickness of constructed blurred segments. Moreover, our approach also applies to disconnected sets which opens perspectives in the study of discrete curves with holes. The tools we used can be extended to 3D and this might lead to decompositions of discrete surfaces into blurred linear patches. 


\section{References}

1. I. Debled-Rennesson, J.-L. Rémy, and J. Rouyer. Segmentation of discrete curves into fuzzy segments. In 9th International Workshop on Combinatorial Image Analysis, volume 12 of Electronic Notes in Discrete Mathematics, 2003.

2. I. Debled-Rennesson, J.-L. Rémy, and J. Rouyer. Segmentation of discrete curves into fuzzy segments, extended version. Technical report, INRIA Report RR-4989, http://www.inria.fr/rrrt/rr-4989.html, 2003.

3. I. Debled-Rennesson and J.P. Reveillès. A linear algorithm for segmentation of digital curves. IJPRAI, 9(6), December 1995.

4. F. Feschet and L. Tougne. Optimal time computation of the tangent of a discrete curve: application to the curvature. In Discrete Geometry and Computer Imagery, Lecture Notes in Computer Science 1568, pages 31-40. Springer Verlag, 1999.

5. M.E. Houle and G.T. Toussaint. Computing the width of a set. IEEE Trans. on Pattern Analysis and Machine Intelligence, 10(5):761-765, 1988.

6. A. Kolesnikov and P. Fränti. Reduced-search dynamic programming for approximation of polygonal curves. Pattern Recognition Letter, 24(14):2243-2254, 2003.

7. M. Lindenbaum and A. Bruckstein. On Recursive, $\mathrm{O}(\mathrm{N})$ Partitioning of a Digitized Curve into Digital Straight Segments. IEEE Transactions on Pattern Analysis and Machine Intelligence, 15(9):949-953, 1993.

8. A. Melkman. On-line Construction of the Convex Hull of a Simple Polygon. Information Processing Letters, 25:11-12, 1987.

9. J. Perez and E. Vidal. Optimum polygonal approximation of digitized curves. Pattern Recognition Letter, 15:743-750, 1994.

10. F.P. Preparata and M.I. Shamos. Computational Geometry: an Introduction. Springer-Verlag, 1985.

11. J.P. Reveillès. Géométrie discrète, calculs en nombres entiers et algorithmique. Thèse d'Etat - Université Louis Pasteur, 1991.

12. A. Rosenfeld and R. Klette. Digital Straightness - a review. Discrete Applied Math., 139(1-3):197-230, 2004.

13. P. L. Rosin. Techniques for assessing polygonal approximations of curves. IEEE Transactions on PAMI, 19(6):659-666, 1997.

14. A.W.M. Smeulders and L. Dorst. Decomposition of discrete curves into piecewise straight segments in linear time. Contemporary Mathematics, 119:169-195, 1991.

15. P. Yin. A tabu search approach to polygonal approximation of digital curves. International Journal of Pattern Recognition and Artificial Intelligence, 14(2):243$255,2000$.

16. D. Zhang and G. Lu. Review of shape representation and description techniques. Pattern Recognition, 37(1):1-19, 2004. 